\title{
Vorwort zur 3., aktualisierten und erweiterten Auflage
}

14 Jahre nach dem Erscheinen dieses Buches erscheint nun eine stärker veränderte Version. Neuere Theorien im Bereich der Grammatik waren zu berücksichtigen. Spracherwerb und Interkulturalität spielen in der aktuellen Diskussion des Fachs und der Öffentlichkeit eine noch größere Rolle und haben in diesem Buch eine stärkere Gewichtung erfahren. Gesellschaftliche Mehrsprachigkeit wird endlich wahrgenommen und die Abkehr von monolingualen Orientierungen steht auch in den Schulen auf der Tagesordnung.

Bachelor-Studiengänge ziehen eine kompaktere Studienweise nach sich, erfordern gleichwohl eine Kenntnis auch der Fachgrundlagen und eigenständiges Erarbeiten von Grundtexten einer Wissenschaft. Eine veränderte Studienorganisation darf nicht dazu führen, den Lernenden Wissen nurmehr in ausgedünnter Form zu servieren. Auch und gerade wer auf Anwendung aus ist, braucht ein solides theoretisches Fundament.

Solche Erfahrungen haben mich als Herausgeber bewogen, das Buch in einigen Teilen umzuarbeiten, damit es seine positiven Wirkungen unverändert weiter entfalten kann. Zum einen wurden die eher didaktischen Texte und der Anteil von Aufgaben verstärkt, damit ein Einsatz in Grundveranstaltungen erleichtert wird. Anderseits bleibt es beim pluralistischen Prinzip und beim Zugang über klassische Texte. Neu aufgenommen wurde etwa Leonard Bloomfield, dessen Buch „Language“ zu den bedeutendsten der Sprachwissenschaft gehört. Es werden gegenwärtig diskutierte Ansätze vermittelt (beispielsweise das Verhältnis von Sprache und Kultur (Tomasello (Kapitel A), Günthner, Heeschen, Rehbein), die Prototypensemantik (Löbner), die Grammatikalisierungsforschung, (Haspelmath) die Optimalitätstheorie (Müller), die Konstruktionsgrammatik (Goldberg, Tomasello (F)).

Ein Reader dieser Art muss in Bewegung bleiben, um im Studium etwas bewegen zu können. Auch das Neue - ob eingestanden oder nicht - beruht auf klassischen Ansätzen. Wer daran die richtigen Fragen und die wissenschaftliche Haltung der Kritik lernt, Zugang zu den Originalen findet, hat viel gewonnen und wird auch die Grenzen des bloß Modischen leichter erkennen.

Der Reader kann mit den grundlegenden Texten (in den Anfangsteilen der Kapitel) zur Einführung genutzt werden. Er kann auch mit den weiteren Texten in Lektürekursen, zur Vertiefung oder zur Prüfungsvorbereitung in höheren Studienphasen eingesetzt werden. Wünschenswert ist, dass der Einstieg durch Lehrende oder Tutorien begleitet wird. Es kann direkt an den Texten 
diskutiert werden, in denen durch das Buch hindurch verschiedene rote Fäden verfolgt werden. Möglich ist auch, dass Kurzpräsentationen (in gemeinsamer Vorbereitung) entstehen, so dass kritische, Thesen und Begründungen herausarbeitende Lektüre und nicht zuletzt die Vermittlung von Ideen und Gedankengängen gelernt werden.

Im Zentrum stehen Leitfragen wie: Was ist Sprache? Ist sie charakteristisch für den Menschen? Wie verhält sie sich zur Natur, wie zur Kultur? Wie ist sie aufgebaut und wie sind Formen und Funktionen aufeinander bezogen? Wie kann das Kind sich eine oder mehrere Sprachen aneignen? Nach welchen Prinzipien funktioniert sprachliche Kommunikation? Inwiefern können wir verstehen, was jemand meint?

Auf meiner Homepage finden sich Materialien, die den Reader ergänzen: http://home.edo.uni-dortmund.de/ hoffmann/Reader.html http://home.edo.uni-dortmund.de/ hoffmann/Biblio.html http://home.edo.uni-dortmund.de/ hoffmann/Links.html

Auch für diese Ausgabe gilt: Ich habe viel von den Dortmunder Studierenden und Doktoranden gelernt, die ich in meinen Dank ebenso einschließe wie Kolleginnen und Kollegen aus dem Fach und Herrn Prof. Dr. Heiko Hartmann und Frau Susanne Rade vom Verlag de Gruyter. Für Hilfe bei den Korrekturen danke ich Jasmin Hirschberg.

Dortmund, Frühling 2010

Ludger Hoffmann 